Article

\title{
Ground-State and Thermodynamical Properties of Uranium Mononitride from Anharmonic First-Principles Theory
}

\author{
Per Söderlind *, Alexander Landa, Aurélien Perron, Babak Sadigh and Tae Wook Heo \\ Lawrence Livermore National Laboratory, 7000 East Ave., Livermore, CA 94550, USA; landa1@1lnl.gov (A.L.); \\ perron1@llnl.gov (A.P.); sadigh1@llnl.gov (B.S.); heo1@llnl.gov (T.W.H.) \\ * Correspondence: soderlind@llnl.gov; Tel.: +1-9254234667
}

Received: 22 August 2019; Accepted: 15 September 2019; Published: 18 September 2019

check for updates

\begin{abstract}
We report on an advanced density-functional theory (DFT) approach for investigating the ground-state and thermodynamical properties of uranium mononitride (UN). The electronic structure for UN at zero temperature is obtained from DFT that utilizes the generalized gradient approximation (GGA) for the electron exchange and correlation functional and includes spin-orbit interaction and an extension with orbital polarization. Thermodynamical properties are computed within the quasi-harmonic approximation in the Debye-Grüneisen model while anharmonicity is captured in the self-consistent ab initio lattice dynamics (SCAILD) scheme. Anharmonic phonons have heretofore never been modeled from first-principles for UN but they turn out to be important. The computed free energy compares well with that of a CALPHAD (CALculation of PHAse Diagrams) assessment of available experimental data.
\end{abstract}

Keywords: density-functional theory; anharmonicity; thermodynamical properties; actinides; uranium mononitride

\section{Introduction}

Uranium mononitride (UN) is an actinide compound that forms in the cubic sodium-chloride structure (B1), similar to other actinide nitrides and carbides. The location of the uranium and nitrogen atoms are interchangeable in this phase that is a face-centered cubic, with one atom in the origin and the other in the cube center. The B1 actinide-nitride and actinide-carbon systems are characterized by high melting temperatures and substantial electrical and thermal conductivities. Sometimes these compounds are magnetic. In the case of UN, the U-U distance is greater $(\sim 3.5 \AA)$ than that of $\alpha$-uranium $(\sim 2.8 \AA)$ and consequently, a magnetic moment forms on the uranium atom in UN but not in $\alpha$-uranium.

Uranium mononitride has received a lot of attention from both experimental [1-29] and computational angles [30-43] due to its potential as a nuclear fuel for fast-breeder reactors [44]. It has superior thermophysical properties, including high melting temperature, uranium loading (content), thermal conductivity, and resistance to corrosion in air and water. It further exhibits good thermal and irradiation behavior, and is compatible with numerous candidate space reactor coolants, e.g., sodium, and other structure materials.

Experimentally, several fundamental properties of UN have been characterized: (i) structural, (ii) mechanical, (iii) thermodynamical, and (iv) magnetic properties. For the structural properties (lattice parameter, density, and thermal expansion) a number of studies involving X-ray diffraction (XRD) have been reported $[1,7,11,16,17,21,22,25,26,29]$. For the mechanical properties (bulk modulus, shear modulus, Poisson's ratio, elastic constants, lattice dynamics), ultrasonic pulse-echo (velocity and frequency) techniques and inelastic neutron scattering have been extensively used and the results from these experiments are presented in $[2,6,7,14,18,21-23,26,27]$. For thermodynamical 
properties (specific heat, enthalpy, entropy, Gibbs free energy, melting point, heat of formation, and the resulting phase-diagram), most of the relevant experiments have relied on specific-heat measurements by different calorimeter capabilities $[4,5,9,10,13,15,19,20,22]$, although the laser flash method is used in [12]. Determinations of the heat of formation for uranium mononitride are reported in References [10,19,20,24]. Recently, Baranov et al. [29] developed an advanced technique to study uranium mononitride fuel thermochemical stability based on XRD, scanning electron microscopy (SEM), and mass-spectrometry. Finally, magnetic properties obtained from neutron scattering have been reported [3].

Accurate modeling of ground-state and particularly thermodynamical properties of uranium mononitride can play an important role in interpreting existing materials data and establishing a better understanding of its thermodynamical stability and fuel performance. Theoretical studies of uranium mononitrides can be divided into one of three categories; phenomenological models, first-principles electronic-structure calculations, and thermodynamical modeling. In this report we focus on the two latter approaches.

We present details of our electronic-structure method in Section 2.1 and its application to investigate phonons in Section 2.2. In the literature, there are numerous papers focused on ab-initio-theory studies of uranium mononitride [30,33-43]. The heat capacity of uranium mononitride has been estimated by molecular-dynamics simulations by Kurosaki et al. [32] utilizing the phonon spectrum data by Baranov et al. [40]. Freyss and Sato [33] calculated the equilibrium lattice parameter, bulk modulus, and cohesive energy. Weck et al. [34] performed all-electron spin-polarized relativistic calculations of the electronic and thermodynamical properties of stoichiometric $\mathrm{UN}$ and $\mathrm{UN}_{2}$ compounds. Modak and Verma [39] studied the electronic properties and phonon-dispersion relations of uranium mononitride using the pseudopotential density-functional-theory (DFT) method.

We note that uranium mononitride is an antiferromagnetic compound (anti-parallel spins on the uranium atoms in 001 planes) with substantial magnetic moments on the uranium atoms $\left(\sim 0.75 \mu_{B}\right)$ [3]. The spin-polarized uranium $5 \mathrm{f}$ electrons in this compound have been treated in different ways in the past. Brooks [30] and others [35,36] employed DFT, while stronger 5f-electron correlations were incorporated by going beyond DFT, applying either electron self-interaction-corrected theory (SIC-DFT) [37] or an on-site Coulomb repulsion term (effective Hubbard U; DFT+U) [38,42,43]. The principal failure of the DFT model is that it favors a ferromagnetic state over the observed anti-ferromagnetic one.

However, the magnetic properties of $\mathrm{UN}$ are not the primary focus of the present study, but rather other ground-state properties that are derived from the energetics of the compound. Moreover, we are specifically interested in the thermodynamical behavior at elevated temperatures near the melting point $(\sim 3000 \mathrm{~K})$. These temperatures are far above the magnetic ordering of uranium mononitride (Néel temperature is $53 \mathrm{~K} \mathrm{[3])} \mathrm{and} \mathrm{therefore} \mathrm{we} \mathrm{have} \mathrm{chosen,} \mathrm{similar} \mathrm{to} \mathrm{[35,36],} \mathrm{to} \mathrm{restrict} \mathrm{ourselves} \mathrm{to} \mathrm{a}$ ferromagnetic configuration for most of our calculations. Another motivation for this simplification is that total energy and bonding properties of ferromagnetic and anti-ferromagnetic UN are nearly indistinguishable, as we shall show below.

The basic idea of the thermodynamical modeling is founded on the CALPHAD (CALculation of PHAse Diagrams) methodology that involves calculating phase equilibria and phase diagrams from experimental inputs [45-47]. Therefore, we employ this method to extract the relevant thermodynamical properties derived from experimental characterizations for comparison with our theory. A general description of the CALPHAD method and its application to the UN system will be presented in Section 2.3.

Due to its potential as a fuel material, knowledge of the thermal behavior is essential. Understanding thermodynamical properties from first-principles theory, including enthalpy of formation, heat capacity, and free energy, is important for reliably determining the phase stability and phase diagram. For the calculation of these properties we go beyond previous first-principles modeling, that are limited to a quasi-harmonic treatment of the lattice vibrations, and here include anharmonic effects via the self-consistent ab initio lattice dynamics (SCAILD) method [48]. This approach is 
computationally efficient, and it accounts for phonon-phonon interactions that may produce strong anharmonic effects. The phonon-phonon coupling was shown to be important for uranium metal [49] but have heretofore been ignored in first-principles modeling for UN.

\section{Computational Methods}

\subsection{Electronic Structure Method}

The results from the electronic-structure calculations presented here come from applying density-functional theory that took shape in the important works by Hohenberg, Kohn, and Sham [50,51]. Density-functional theory is in principle correct, but it depends on practical simplifications. The most important one is the treatment of the electrons exchange and correlation interactions. For these interactions many different approximations have been proposed. Here we are applying the generalized gradient approximation (GGA) that is better for the actinides than the previously used local density approximation [52] and even some more recent formulations [53]. In addition to spin-orbit interaction we extend DFT to include effects of orbital-orbital interactions as we will discuss below.

In terms of the technical details of the calculations, we are applying two approaches. For all calculations, except those related to the self-consistent phonon method (see below), we utilize an all-electron full-potential linear muffin-tin orbital (FPLMTO) method that is well established [54] and has been described in detail [55]. In this method, no approximations are made for the electron core states that lie deeper in energy than the valence states, unlike the pseudopotential method. The core approximation made in the latter technique is computationally efficient but can sometimes cause inaccuracies that are avoided in all-electron calculations.

The present implementation does not make any assumptions beyond that of the electron exchange and correlation functional. Basis functions, electron densities, and potentials are calculated without any geometrical approximation and these are expanded in spherical harmonics (with a cut-off $l_{\max }=8$ ) inside non-overlapping (muffin-tin) spheres surrounding each atom and in Fourier series in the region between these muffin-tin spheres. There is a choice how to define the length of the muffin-tin sphere radius ( $\mathrm{r}_{\mathrm{MT}}$ ) and here it is chosen so that $\mathrm{r}_{\mathrm{MT}} / \mathrm{r}_{\mathrm{WS}} \sim 0.8$, where $\mathrm{r}_{\mathrm{WS}}$ is the Wigner-Seitz (atomic-sphere) radius. The radial part of the basis functions inside the muffin-tin spheres are calculated from a wave equation for the $l=0$ component of the potential that include all relativistic corrections including spin-orbit coupling for $\mathrm{d}$ and $\mathrm{f}$ states but not for the $\mathrm{p}$ states, following the arguments in Reference [56]. Likewise, orbital-orbital interaction (orbital polarization, $\mathrm{OP}$ ) is only considered for $\mathrm{d}$ and $\mathrm{f}$ states. The formulation of the orbital polarization is obtained from Eriksson, Brooks, and Johansson [57] and this interaction has been shown to be important for actinides, particularly plutonium [58].

For the two-atom per cell UN structure we applied $500 \mathrm{k}$ points in the irreducible part of the Brillouin zone and each energy eigenvalue was broadened with a Fermi-Dirac distribution $(\mathrm{T}=300 \mathrm{~K})$. Uranium mononitride is an anti-ferromagnet but within the GGA a ferromagnetic ordering has lower energy and we chose to adopt ferromagnetism for most calculations. However, the energetics and bonding are very similar for both and close to experiment [21], see Table 1, and the actual choice (ferromagnetic or anti-ferromagnetic) does likely not matter much for the properties we focused on here. The reported atomic volume, bulk modulus and its pressure derivative in Table 1 are obtained from a Vinet fit [59] to the calculated DFT pressures.

For the self-consistent phonon method (see below) we applied a 54-atom UN cell in the calculations of the forces that are required for this approach. As this task is computationally rather challenging, we utilized a more efficient electronic-structure code with the additional simplifications of ignoring magnetic ordering and spin-orbit interaction. Hence, we employed the Vienna ab initio simulation package VASP [60-62]. Here, the projected augmented wave (PAW) potentials have a $450 \mathrm{eV}$ energy cut off and we applied a k point mesh on a $2 \times 2 \times 2$ Monkhorst-Pack grid. 
Table 1. Uranium mononitride (UN) results from density-functional-theory (DFT) ferromagnetic (FM), anti-ferromagnetic (AF), non-magnetic (NM) calculations and experimental data $[3,20,21] . \mathrm{V}, \mathrm{B}$, and $\mathrm{B}^{\prime}$ are the atomic volume, bulk modulus, and the bulk modulus pressure derivative $\left(\mathrm{B}^{\prime}=\mathrm{dB} / \mathrm{dP}\right)$, respectively. $\Delta \mathrm{E}$ is the relative total energy and $\mathrm{M}$ the total (spin and orbital) magnetic moment on the uranium atom. All results are obtained from calculations that include orbital polarization except for the magnetic moment in parenthesis that refers to a treatment with spin-orbit coupling only. $\Delta_{\mathrm{f}} \mathrm{H}$ is the formation enthalpy.

\begin{tabular}{ccccccccc}
\hline Method & $\begin{array}{c}\mathbf{V} \\
\left(\AA^{3}\right)\end{array}$ & $\begin{array}{c}\mathbf{B} \\
(\mathbf{G P a})\end{array}$ & $\mathbf{B}^{\prime}$ & $\begin{array}{c}\Delta \mathrm{E} \\
(\mathbf{m R y} / \mathbf{a t})\end{array}$ & $\begin{array}{c}\Delta \mathrm{E} \\
(\mathbf{k J} / \mathbf{m o l})\end{array}$ & $\begin{array}{c}\mathbf{M} \\
\left(\mu_{\mathbf{B}}\right)\end{array}$ & $\begin{array}{c}\boldsymbol{\Delta}_{\mathbf{f}} \mathbf{H} \\
(\mathbf{m R y} / \mathbf{a t})\end{array}$ & $\begin{array}{c}\boldsymbol{\Delta}_{\mathbf{f}} \mathbf{H} \\
(\mathbf{k J} / \mathbf{m o l})\end{array}$ \\
\hline DFT-FM & 14.85 & 182 & 5.2 & 0 & 0 & $0.78(1.56)$ & -226.6 & -297.5 \\
DFT-AF & 14.85 & 183 & 5.0 & 1.5 & 1.97 & 0.78 & $\mathrm{n} / \mathrm{a}$ & $\mathrm{n} / \mathrm{a}$ \\
DFT-NM & 14.75 & 232 & 2.0 & 2.3 & 3.02 & 0 & $\mathrm{n} / \mathrm{a}$ & $\mathrm{n} / \mathrm{a}$ \\
Expt & 14.62 & $191-206$ & 6.3 & $\mathrm{n} / \mathrm{a}$ & $\mathrm{n} / \mathrm{a}$ & 0.75 & -223.6 & -293.6 \\
\hline
\end{tabular}

\subsection{Self-Consistent Ab Initio Lattice Dynamics Method (SCAILD)}

The approach is described in detail [63] and not repeated here, but the general idea is to account for thermal vibrations of the atoms and their interactions. This is accomplished by (i) calculating the forces on atoms, displaced from the ideal positions according to the temperature, and (ii) computing the phonon density of states (DOS). As the forces, displacements, and the phonon DOS depend on each other the scheme is set up to self-consistently determine the temperature dependence of the lattice dynamics. We conducted SCAILD calculations, utilizing a 54-atom cell for UN, for five temperatures, $\mathrm{T}=1000,1500,2000,2500$, and $3000 \mathrm{~K}$. In addition, we considered three strategically chosen atomic volumes for a total of 15 simulations. The lattice free energy is well converged with 105 SCAILD iterations for each simulation and the result was used to evaluate the total free energy (also adding DFT electronic contributions) as a function of temperature at ambient pressure. In Figure 1, we illustrate these lattice vibration energies (here at constant atomic volume, $14.85 \AA^{3}$ ) as functions of number of SCAILD iterations. The method was also used to evaluate, from the phonon DOS, the heat capacity that can be compared to experimental data.

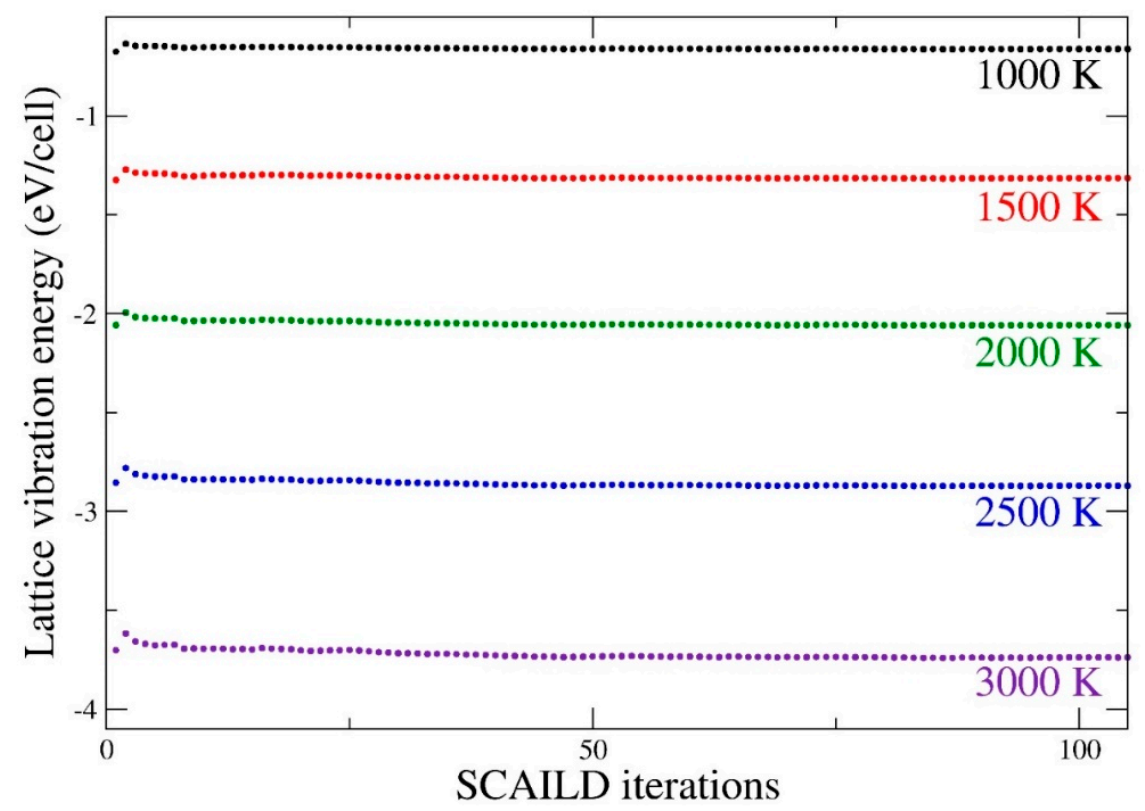

Figure 1. Self-Consistent $\mathrm{Ab}$ Initio Lattice Dynamics (SCAILD) lattice vibration energies for five temperatures, at constant atomic volume $\left(14.85 \AA^{3}\right)$, as functions of number of SCAILD iterations. 


\subsection{CALPHAD Method}

The CALPHAD method was employed in this study to extract the reliable experimental thermodynamic properties for comparison with our computed results, which validated our theoretical approach. In general, the major function of the CALPHAD approach is to model the Gibbs energy and establish a phase diagram [45-47]. CALPHAD is an iterative method that adjusts the parameters that describes the Gibbs energy functions of various phases in a system in order to construct a phase diagram that best reproduces the available experimental thermodynamical and phase-diagram data. Once such functions have been assessed, they are compiled in a database that can be used for computational thermodynamical predictions of multi-component systems.

In addition to obvious comparison between the existing CALPHAD data and the theoretical calculations, we emphasize that the coupling of CALPHAD and ab initio calculations enhances the thermodynamic modeling capability when material systems with many thermodynamic unknowns are considered. First of all, ab initio calculations (e.g., the heats of formation and transformation) can directly provide essential inputs to the CALPHAD modeling framework when experimental data are sparse or missing. Second, it is worth noting that the process of parameter optimization and error minimization involved in the CALPHAD method is an inverse problem with infinite degrees of freedom [64]. Thus, many possible combinations of values of user-defined parameters could produce phase boundaries and diagrams that perfectly coincide. Therefore, the use of ab initio energetics during the CALPHAD assessment constrains the optimization process and validates the resulting thermodynamical database, not only from a diagrammatic basis, but also from a reliable energetics basis. Examples of ab-initio-informed CALPHAD assessments for actinide systems can be found in $[65,66]$. Following the present work, ab initio predictions of specific heat and free energy versus temperature for actinide systems is extremely useful to the CALPHAD community.

The CALPHAD thermodynamical modeling of the U-N binary system was initially performed by Chevalier et al. [31] from a critical assessment of most of the available experimental information on this system. The agreement between experimental and calculated phase diagram or thermodynamical properties was quite satisfactory. However, Besmann et al. [67] mentioned that the calculated UN decomposition nitrogen pressure versus temperature was significantly lower than almost all reported measurements. Thus, Besmann [67] adjusted the UN Gibbs free energy by $+12 \mathrm{~kJ} / \mathrm{mol}$ from that of Chevalier et al. [31] to bring the calculated pressures into relative agreement with reported measurements. This last CALPHAD assessment of the UN Gibbs free energy is considered in the present study.

\section{Results}

Uranium mononitride is a rather stable compound and that is reflected in a strongly negative formation enthalpy; $-293.6 \mathrm{~kJ} / \mathrm{mol}(-223.65 \mathrm{mRy} / \mathrm{at})$ [20]. The formation enthalpy can be modeled within the DFT approach because the energy of the constituents of UN (uranium and nitrogen) as well as the compound itself (ignoring the possibility of a non-stochiometric compound) can be calculated. Uranium metal forms in an orthorhombic structure, $\alpha$ uranium, that was studied within the DFT model a while ago [68]. The structure is defined by axial b/a and c/a ratios as well as an internal parameter, $y$, that were carefully determined [68] and these parameters were used in the present calculation for $\alpha$-uranium. Uranium mononitride, on the other hand, forms in the cubic sodium-chloride structure and has no free parameters due to its symmetry. The calculation of the nitrogen (nitrogen gas, $\mathrm{N}_{2}$ ) is less straightforward in the context of conventional density-functional theory. Sedmidubský et al. [69] addressed the problem associated with $\mathrm{N}_{2}$ gas molecules in their attempts to calculate formation enthalpies of the actinide mononitrides from DFT. They simulated an $\mathrm{N}_{2}$ dimer by constructing a large tetragonal unit cell and compared its energy with nitrogen atoms placed in a cubic cell and very far apart. From this they deduced a dissociation energy of $\mathrm{N}_{2}=462.2 \mathrm{~kJ} / \mathrm{mol}$ ( $\left.352.08 \mathrm{mRy} / \mathrm{at}\right)$. In the present study we added this amount to the energy of a large cell of the simple cubic $\alpha-\mathrm{N}_{2}$ [70] for an estimate of the nitrogen-atom energy. Combining this result and the calculated energies of $\alpha$ - $U$ and 
$\mathrm{UN}$, we obtained a formation enthalpy of $\mathrm{UN} ; \Delta_{\mathrm{f}} \mathrm{H}=-297.5 \mathrm{~kJ} / \mathrm{mol}(-226.6 \mathrm{mRy} / \mathrm{at})$, in exceptional agreement with the experimental value of $-293.6 \mathrm{~kJ} / \mathrm{mol}(-223.6 \mathrm{mRy} / \mathrm{at})$ [20].

In Table 1 we collect formation enthalpy and some other calculated ground-state properties for UN. Overall, our results are in excellent agreement with experiment and depend only very weakly on the magnetic configuration. Even the non-magnetic treatment appears reasonable, suggesting that ignoring magnetism, as is done here for the phonon calculations, is sensible. The calculated magnetic moment agrees with neutron diffraction [3] only when orbital polarization is considered. Without orbital-orbital coupling the orbital moment is severely underestimated and the total moment too large (the spin and orbital moments couple anti-parallel).

In addition to thermodynamical stability, mechanical stability is important for a relevant material. The elastic constants provide information on mechanical properties and they have been measured [18] and computed [39,71] for UN and we compare our results to those in Table 2. In general, DFT elastic constants have larger errors than either the atomic volume or the bulk modulus and with that in mind, the calculated zero-temperature elastic constants compare very favorably with the measured room-temperature data.

Table 2. Calculated, present, and published [39,71], and experimental [18] elastic constants for UN.

\begin{tabular}{cccc}
\hline Method & $\mathbf{C}_{\mathbf{1 1}}(\mathbf{G P a})$ & $\mathbf{C}_{\mathbf{1 2}}$ (GPa) & $\mathbf{C}_{\mathbf{4 4}}$ (GPa) \\
\hline DFT-FM (present) & 390 & 77.9 & 79.6 \\
DFT-GGA [39] & 428 & 136.6 & 29.7 \\
DFT-GGA [71] & 404.6 & 124.2 & 45.0 \\
Expt & 391 & 90 & 80 \\
\hline
\end{tabular}

Reviewing the performance of the DFT (GGA+OP) model for UN in Tables 1 and 2, we realize that there is no compelling argument for going beyond this level of theory. In a recent DFT+U study [38] the atomic volume and bulk modulus were calculated as functions of the Hubbard $U$ parameter for the purpose of finding the $U$ value that best reproduces the data $(U=2 \mathrm{eV})$. However, for the optimized value of $U$, the agreement with experiment was still worse than our GGA+OP approach presented in Table 1 . The reason the DFT $+\mathrm{U}$ result was less accurate is likely that the electronic structure was not obtained from an all-electron treatment and that neither spin-orbit coupling, nor orbital polarization was accounted for [38].

As discussed above, being a potential fuel, the thermodynamical properties of uranium mononitride at rather high temperatures are of interest. At lower to moderate temperatures one expects a quasi-harmonic approximation of the lattice vibrations to be sufficient [38] while at temperatures well above $1000 \mathrm{~K}$ anharmonic effects become increasingly relevant. With the self-consistent phonon approach, it is possible to address strong phonon-phonon coupling that leads to anharmonic lattice vibrations that go beyond the quasi-harmonic behavior.

In Figure 2 we show the free energy for UN obtained from SCAILD. Results from only the lattice-vibration contribution, at a fixed $14.85 \AA^{3}$ atomic volume, are displayed with open squares. When adding the electron contribution, including the electronic entropy, the free energy drops somewhat (solid circles). Finally, evaluating the free-energy contributions at zero pressure (minimizing the free energy with respect to volume), results in the solid squares in Figure 2. For comparison we also show (solid line) the results from a CALPHAD assessment of available experimental data. Clearly, the first-principles treatment is consistent with the CALPHAD result, suggesting a rather accurate theoretical model. 


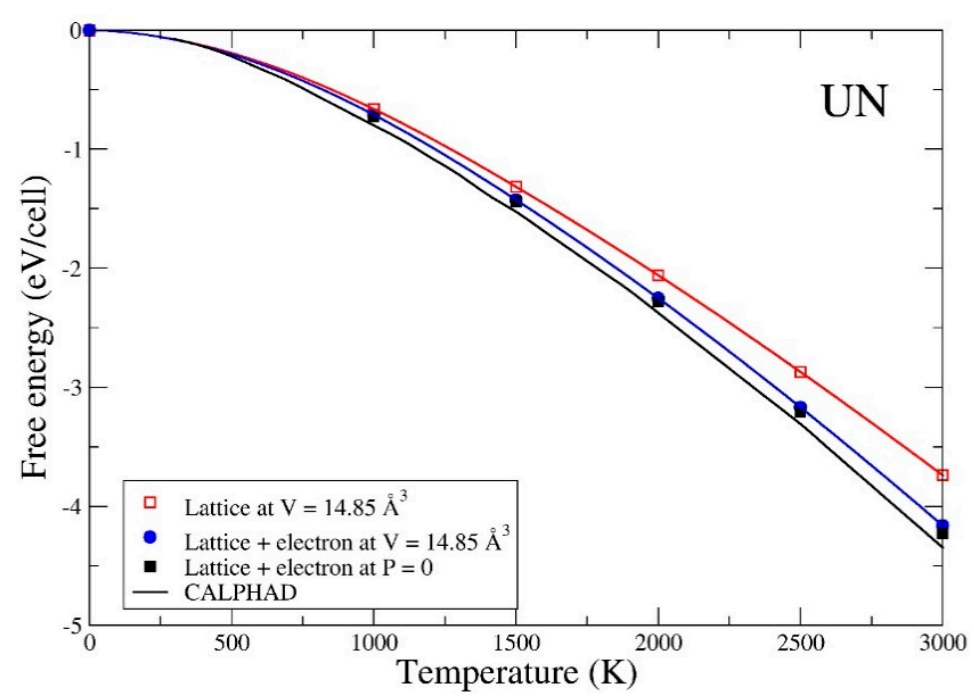

Figure 2. DFT and CALPHAD (from $300 \mathrm{~K}$ ) free energies for UN. Open squares refer to SCAILD lattice energies at constant atomic volume $\left(14.85 \AA^{3}\right)$. The DFT electron free energy that includes electronic entropy was added for the solid circles. The solids squares refer to results from volume relaxation (pressure is zero) that includes both electronic and lattice contributions. The lines connecting the symbols are guides to the eye only. The solid line without symbols refers to our CALPHAD results.

In addition to the free energy, it is interesting to directly contrast our calculated heat capacity with experimental data. In Figure 3 we show two sets of calculations, one assuming quasi-harmonic lattice vibrations, acquired from the GIBBS2 package [72-75], and the other, allowing for anharmonic phonons, determined by the SCAILD method. For both these sets we add the electronic contribution obtained from the electronic structure. The $C_{v}$ data from SCAILD were converted to $C_{p}$ by applying the difference in these quantities computed by GIBBS2. There are also numerous sets of experimental data $[4,7,8,12,13,15,19]$ included in the figure. The anharmonic phonon model compares very well with available experimental data except those from [7] that appears to deviate from all other experimental data above $1700 \mathrm{~K}$. The quasi-harmonic model does reasonably well at lower temperatures up to $1000 \mathrm{~K}$ but beyond that it is insufficient for reproducing the trend of the experimental data.

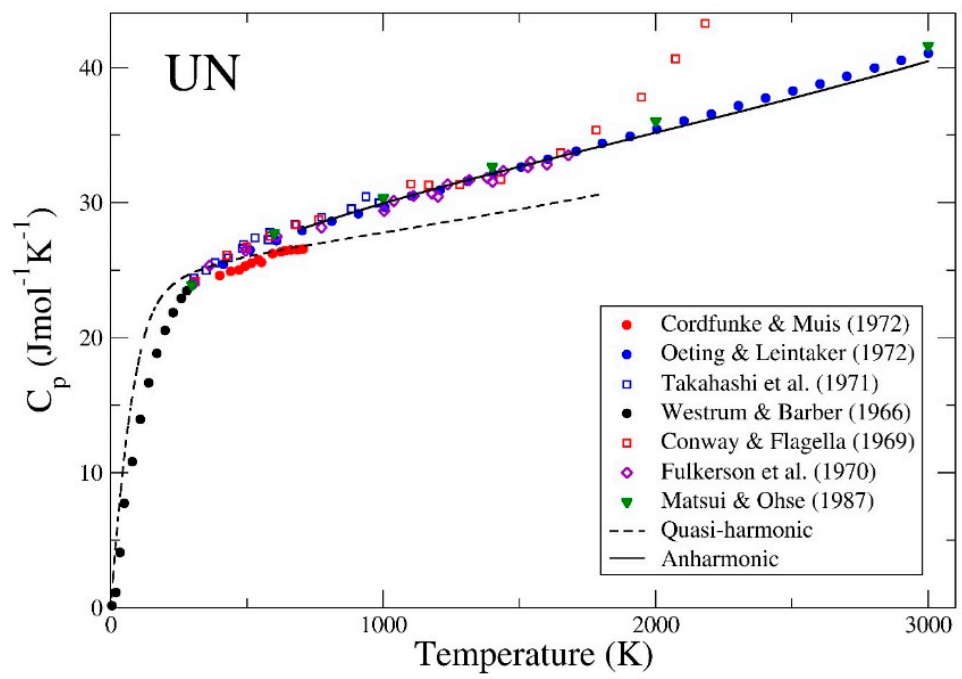

Figure 3. Theoretical models for the heat capacity at ambient pressure, assuming quasi-harmonic and anharmonic phonons. The experimental data are collected from References $[4,7,8,12,13,15,19]$. Note, the heat capacity is calculated per gram-atom (g-at.) of $\mathrm{N}_{2}$ and $1 \mathrm{~mol}$ of $\mathrm{N}_{2}$, which is equivalent to 1 gram-molecule of $\mathrm{N}_{2}$, contains 2 gram-atoms of $\mathrm{N}_{2}$. 
The Debye temperature is another quantity for which the models can be tested against experiments. The phonon spectrum of UN is known from neutron scattering [76] and from this spectrum a Debye temperature $\Theta_{D}=181 \mathrm{~K}$ can be deduced [40]. Our anharmonic treatment of UN predicts $\Theta_{D}=190 \mathrm{~K}$ with only a very weak temperature dependence, while our quasi-harmonic treatment (GIBBS2) results in a substantially larger $\Theta_{\mathrm{D}}=269 \mathrm{~K}$ in worse agreement with neutron scattering. The failure of the quasi-harmonic model to accurately predict the Debye temperature for UN underscores the importance of anharmonic effects in this compound.

\section{Summary and Conclusions}

We applied a DFT-GGA model, including orbital polarization, for uranium mononitride and found that for thermodynamical and mechanical properties, the approach is appropriate. The formation enthalpy as well as equilibrium volume, bulk modulus, and elastic constants all agree very favorably with experimental data. The electronic-structure model does not reproduce the observed anti-ferromagnetic ground state but rather a ferromagnetic state. However, we show that the energetics depends very weakly on the actual magnetic configuration and for most calculations we thus assume ferromagnetic order. We extend DFT to include orbital polarization, and this interaction is particularly important for the magnetic properties.

For the high-temperature calculations, we simplified the treatment of the electronic structure and overlooked magnetic effects and spin-orbit coupling. We are, to some extent, justified in this simplification because magnetic order is absent above the ordering temperature and due to the thermal broadening, we expect the effect of spin-orbit interaction to greatly diminish. These electron correlations can in principle be addressed in the modeling of the phonons, but this is currently beyond our computational capabilities.

Our phonon calculations above $1000 \mathrm{~K}$ show that accurate behavior of the free energy as well as heat capacity is only achieved when anharmonic lattice dynamics is considered. Specifically, for the free energy, our present ab initio model is consistent with the CALPHAD assessment of the known experimental data. In addition, the agreement between the anharmonic model and the corresponding measured data is excellent for the heat capacity and Debye temperature, demonstrating the importance of the anharmonic phonons.

Author Contributions: P.S. and A.L. conceived the study. P.S. performed DFT and anharmonic calculations. A.L. performed quasi-harmonic calculations. A.P. performed CALPHAD assessments. All authors helped to write the paper.

Funding: This research received no external funding.

Acknowledgments: We thank L. Yang, J. Jeffries, and K. Holliday for helpful discussions. This work was performed under the auspices of the U.S. DOE by LLNL under Contract DE-AC52-07NA27344 and was partly funded by the Laboratory Directed Research and Development Program at LLNL under Project Tracking Codes No. 11-ER-033 and 18-SI-001.

Conflicts of Interest: The authors declare no conflict of interest.

\section{References}

1. Kempter, C.P.; Elliott, R.O. Thermal expansion of $\langle\mathrm{UN}\rangle,\left\langle\mathrm{UO}_{2}>,\left\langle\mathrm{UO}_{2} \bullet \mathrm{ThO}_{2}\right\rangle\right.$, and $\left\langle\mathrm{ThO}_{2}>\right.$. J. Chem. Phys. 1959, 30, 1524-1526. [CrossRef]

2. Speidel, E.O.; Keller, D.J. Fabrication and Properties of Hot-Pressed Uranium Mononitride; BMI-1633; Battelle Memorial Institute: Columbus, OH, USA, 1963. [CrossRef]

3. Curry, N.A. An investigation of the magnetic structure of uranium nitride by neutron diffraction. Proc. Phys. Soc. 1965, 86, 1193-1198. [CrossRef]

4. Westrum, E.G.; Barber, C.M. Uranium mononitride: Heat capacity and thermodynamic properties from 5 to 350 K. J. Chem. Phys. 1966, 45, 635-638. [CrossRef] 
5. Councell, J.F.; Dell, R.M.; Martin, J.F. Thermodynamic properties of uranium compounds. Part 2.-Low-temperature heat capacity and entropy of three uranium nitrides. Trans. Faraday Soc. 1966, 62, 1736-1747. [CrossRef]

6. Padel, A.; De Novion, C.H. Constantes elastiques des carbides, nitrides et oxydes d'uranium ed de plutonium. J. Nucl. Mater. 1969, 33, 40-51. [CrossRef]

7. Conway, J.B.; Flagella, P.N. Physical and Mechanical Properties of Reactor Materials; GEMP-1012; General Electric Company: Cincinnati, OH, USA, 1969.

8. Fulkerson, W.; Kollie, T.G.; Weawer, S.C.; Moore, J.P.; Williams, R.K. Plutonium 1970 and other Actinides Part I and II. In Proceedings of the 4th International Conference on Plutonium and Other Actinides (AIME), Santa Fe, NM, USA, 5-9 October 1970.

9. Affortit, C. Chaleur specifique de UC et UN. J. Nucl. Mater. 1970, 34, 105-107. [CrossRef]

10. Akhachinskii, V.V.; Bashlykov, S.N. Thermodynamics of the uranium-carbon, uranium-nitrogen, and plutonium-carbon systems. Atom. Enegriya 1970, 29, 1211-1219. [CrossRef]

11. Benz, R.; Balog, G.; Baca, B.H. U-UO2-UN2 phase diagram. High Temp. Sci. 1994, 2, 405-406. [CrossRef]

12. Takahashi, Y.; Murabayashi, M.; Akimoto, Y.; Mukaibo, T. Uranium mononitride: Heat capacity and thermal conductivity from 298 to 1000 K. J. Nucl. Mater. 1971, 38, 303-308. [CrossRef]

13. Cordfunke, E.H.P.; Muis, R.P. The heat capacity of uranium mononitride. J. Nucl. Mater. 1972, 42, $233-234$. [CrossRef]

14. Guinan, M.; Cline, C.F. Elastic properties of uranium mononitride at 298 K. J. Nucl. Mater. 1972, 43, $205-206$. [CrossRef]

15. Oeting, F.L.; Leitnaker, J.M. The chemical thermodynamic properties of nuclear materials I. uranium mononitride. J. Chem. Thermodyn. 1972, 4, 199-211. [CrossRef]

16. Muromura, T.; Tagawa, H. Lattice parameter of uranium mononitride. J. Nucl. Mater. 1979, 79, $264-266$. [CrossRef]

17. Benedict, U. Study of actinide metals and actinide compounds under high pressures. J. Less Common Met. 1984, 100, 153-170. [CrossRef]

18. Jackman, J.A.; Holden, T.M.; Buyers, W.J.L.; De Villiers Du Plessis, P.; Vogt, O.; Genossar, J. Systematic study of the lattice dynamics of the uranium rocksalt-structure compounds. Phys. Rev. B 1986, 33, 7144-7153. [CrossRef]

19. Matsui, T.; Ohse, R.W. Thermodynamic properties of uranium nitride, plutonium, nitride and uranium-plutonium mixed nitride. High Temp. High Press. 1987, 18, 1-17.

20. Krikorian, O.H. Thermal expansivity correlations for refractory materials with the NaCl-type structure. High Temp. High Press. 1988, 20, 169-175.

21. Staun Olsen, J.; Gerward, L.; Benedict, U.; Dabos, S.; Itié, J.-P.; Vogt, O. Bulk moduli and high-pressure phases of the uranium rocksalt structure compounds: II. The monopnictides. High Press. Res. 1989, 1, 253-266. [CrossRef]

22. Hayes, S.L.; Thomas, J.K.; Peddicord, K.L. Material property correlations for uranium mononitride: I. Physical properties. J. Nucl. Mater. 1990, 171, 262-318. [CrossRef]

23. Benedict, U. Comparative aspects of the high-pressure behaviour of lanthanide and actinide compounds. J. Alloys Compd. 1995, 223, 216-225. [CrossRef]

24. Wang, W.E.; Olander, D.R. Thermodynamics of gas dissolution in liquid metals with extensive solubility; the $\mathrm{U}(\mathrm{L})-\mathrm{N}, \mathrm{Zr}(\mathrm{L})-\mathrm{O}$, and Th(L)-O systems. J. Alloys Compd. 1995, 228, 31-36. [CrossRef]

25. Le Bihan, T.; Idiri, M.; Heathman, S. New investigation of pressure-induced rhombohedral distortion of uranium nitride. J. Alloys Compd. 2003, 358, 120-125. [CrossRef]

26. Muta, H.; Kurosaki, K.; Masayoshi, U. Thermal and mechanical properties of uranium nitride prepared by SPS technique. J. Mater. Sci. 2008, 43, 6429-6434. [CrossRef]

27. Aczel, A.A.; Granroth, G.E.; MacDougall, G.J.; Buyers, W.J.L.; Abernathy, D.L.; Samolyuk, G.D.; Sticks, G.M.; Nagler, S.E. Quantum oscillations of nitrogen atoms in uranium nitride. Nat. Commun. 2012, 3, 1124. [CrossRef] [PubMed]

28. King, D.M.; Tuna, F.; McInnes, E.J.L.; McMaster, J.; Lewis, W.; Blake, A.J.; Liddle, S.T. Synthesis and structure of a terminal uranium nitride complex. Science 2012, 337, 717-720. [CrossRef] [PubMed] 
29. Baranov, V.G.; Tenishev, A.V.; Kuzmin, R.S.; Pokrovskiy, S.A.; Mikhachik, V.V.; Astafyev, V.A.; Taubin, M.L.; Solntseva, E.S. Thermal stability investigation technique for uranium nitride. Ann. Nucl. Energy 2016, 87, 784-792. [CrossRef]

30. Brooks, M.S.S. Electronic structure of NaCl-type compound of the light actinides. III. The actinide nitride series. J. Phys. F Met. Phys. 1984, 14, 857-871. [CrossRef]

31. Chavalier, P.-Y.; Fisher, E.; Cheynet, B. Thermodynamic modelling of the N-U system. J. Nucl. Mater. 2000, 280, 136-150. [CrossRef]

32. Kurosaki, K.; Yano, K.; Yamada, K.; Uno, M.; Yamanaka, S. A molecular dynamics study of the heat capacity of uranium mononitride. J. Alloys Compd. 2000, 297, 1-4. [CrossRef]

33. Freyss, M.; Sato, I. Ab initio study of uranium and plutonium carbides and nitrides. In Recent Advances in Actinide Science; Alvarez, R., Bryan, N.D., May, I., Eds.; RSC Publishing: Cambridge, UK, 2005; pp. 430-432.

34. Weck, P.F.; Kim, E.; Balakrishnan, N.; Poineau, F.; Yeamans, C.B.; Czerwinski, K.R. First-principles study of single-crystal uranium mono- and dinitride. Chem. Phys. Lett. 2007, 443, 82-86. [CrossRef]

35. Evarestov, R.A.; Losev, M.V.; Panin, A.I.; Mosyagin, N.S.; Titov, A.V. Electronic structure of crystalline uranium nitride: LCAO DFT calculations. Phys. Stat. Sol. (b) 2008, 245, 114-122. [CrossRef]

36. Evarestov, R.A.; Panin, A.I.; Bandura, A.V.; Losev, M.V. Electronic structure of crystalline uranium nitrides $\mathrm{UN}, \mathrm{U}_{2} \mathrm{~N}_{3}$ and $\mathrm{UN}_{2}$ : LCAO calculations with the basis set optimization. J. Phys. Conf. Ser. 2008, 117, 012015. [CrossRef]

37. Petit, L.; Svane, A.; Szotek, Z.; Temmerman, W.M.; Stocks, G.M. Ground-state electronic structure of actinide monocarbides and mononitrides. Phys. Rev. B 2009, 80, 045124. [CrossRef]

38. Lu, Y.; Wang, B.-T.; Li, R.-W.; Shi, H.; Zhang, P. Structural, electronic, and thermodynamic properties of UN: Systematic density functional calculations. J. Nucl. Mater. 2010, 406, 218-222. [CrossRef]

39. Modak, P.; Verma, A.K. First-principles investigation of electronic, vibrational, elastic, and structural properties of ThN and UN up to $100 \mathrm{GPa}$. Phys. Rev. B 2011, 84, 024108. [CrossRef]

40. Baranov, V.G.; Devyatko, Y.N.; Tenishev, A.V.; Khlunov, A.V.; Khomyakov, O.V. A physical model for evaluating uranium nitride specific heat. J. Nucl. Mater. 2013, 443, 248-251. [CrossRef]

41. Su, Q.; Deng, H.; Ao, B.; Xiao, S.; Li, X.; Chen, P.; Hu, W. First-principles study of the interaction of nitrogen atom with $\alpha$-uranium: From surface absorption to bulk diffusion. J. Appl. Phys. 2014, 115, 164902. [CrossRef]

42. Claisse, A.; Klipfel, M.; Lindbom, N.; Freyss, M.; Olsson, P. GGA+U study of uranium mononitride: A comparison of the U-ramping and occupation matrix scheme and incorporation energies of fission products. J. Nucl. Mater. 2016, 478, 119-124. [CrossRef]

43. Zhou, D.; Yu, J.-H.; Pu, C.; Song, Y. Prediction of stable ground-state uranium nitrides at ambient and high pressures. arXiv 2018, arXiv:1804.00095v1.

44. Streit, M.; Franz, I. Nitrides as a nuclear fuel option. J. Eur. Ceram. Soc. 2005, 25, 2687-2692. [CrossRef]

45. Kaufman, L.; Bernstein, H. Computer Calculation of Phase Diagrams with Special Reference to Refractory Metals; Academic Press: New York, NY, USA, 1970.

46. Saunders, N.; Miodownik, A. CALPHAD Calculation of Phase Diagrams: A Comprehensive Guide; Pergamon materials series; Elsevier Science: Amsterdam, The Netherlands, 1998.

47. Lukas, H.; Fries, S.; Sundman, B. Computational Thermodynamics: The CALPHAD Method; Cambridge University Press: Cambridge, UK, 2007.

48. Souvatzis, P.; Eriksson, O.; Katsnelson, M.I.; Rudin, S.P. Entropy driven stabilization of energetically unstable crystal structures explained from first principles theory. Phys. Rev. Lett. 2008, 100, 095901. [CrossRef] [PubMed]

49. Söderlind, P.; Grabowski, B.; Yang, L.; Landa, A.; Björkman, T.; Souvatzis, P.; Eriksson, O. High-temperature stabilization of $\gamma$-uranium from relativistic first-principles theory. Phys. Rev. B 2012, 85, 060301. [CrossRef]

50. Hohenberg, P.; Kohn, W. Inhomogeneous electron gas. Phys. Rev. 1964, 136, B864-B871. [CrossRef]

51. Kohn, W.; Sham, L. Self-consistent equations including exchange and correlation effects. Phys. Rev. 1965, 140, A1133-A1138. [CrossRef]

52. Söderlind, P.; Eriksson, O.; Johansson, B.; Wills, J.M. Electronic properties of f-electron metals using the generalized gradient approximation. Phys. Rev. B 1994, 50, 7291-7294. [CrossRef] [PubMed]

53. Söderlind, P.; Gonis, A. Assessing a solids-biased density-gradient functional for actinide metals. Phys. Rev. B 2010, 82, 033102. [CrossRef] 
54. Lejaeghere, K.; Bihlmayer, G.; Björkman, T.; Blaha, P.; Blügel, S.; Blum, V.; Caliste, D.; Castelli, I.E.; Clark, S.J.; Dal Corso, A.; et al. Reproducibility in density functional theory calculations for solids. Science 2016, 351, aad3000(1)-aad3000(7). [CrossRef]

55. Wills, J.M.; Alouani, M.; Andersson, P.; Delin, A.; Eriksson, O.; Grechnyev, O. Full-Potential Electronic Structure Method; Springer Series in Solid-State Science; Springer: Berlin/Heidelberg, Germany, 2010; Volume 167.

56. Nordström, L.; Wills, J.M.; Andersson, P.H.; Söderlind, P.; Eriksson, O. Spin-orbit coupling in the actinide elements: A critical evaluation of theoretical equilibrium volumes. Phys. Rev. B 2000, 63, 035103. [CrossRef]

57. Eriksson, O.; Brooks, M.S.S.; Johansson, B. Orbital polarization in narrow-band systems: Application to volume collapses in light lanthanides. Phys. Rev. B 1990, 41, 7311-7314. [CrossRef]

58. Söderlind, P.; Landa, A.; Sadigh, B. Density-functional theory for plutonium. Adv. Phys. 2019, 68, 1-47. [CrossRef]

59. Vinet, P.; Ferrante, J.; Smith, J.R.; Rose, J.H. Universal equation of state for solids. J. Phys. C Solid State Phys. 1986, 19, L467-L473. [CrossRef]

60. Kresse, G.; Hafner, J. Ab initio molecular dynamics for liquid metals. Phys. Rev. B 1993, 47, 558-561. [CrossRef] [PubMed]

61. Kresse, G.; Hafner, J. Ab initio molecular-dynamics simulation of the liquid-metal-amorphous-semiconductor transition in germanium. Phys. Rev. B 1994, 49, 14251-14269. [CrossRef] [PubMed]

62. Kresse, G.; Joubert, D. From ultrasoft pseudopotentials to the projector augmented-wave method. Phys. Rev. B 1999, 59, 1758-1775. [CrossRef]

63. Souvatzis, P.; Eriksson, O.; Katsnelson, M.I.; Rudin, S.P. The self-consistent ab initio lattice dynamical method. Comput. Mat. Sci. 2009, 44, 888-894. [CrossRef]

64. Bajaj, S.; Landa, A.; Söderlind, P.; Turchi, P.E.A.; Arroyave, R. The U-Ti system: Strengths and weaknesses of the CALPHAD method. J. Nucl. Mater. 2011, 419, 177-185. [CrossRef]

65. Perron, A.; Turchi, P.E.A.; Landa, A.; Söderlind, P.; Ravat, B.; Oudot, B.; Delaunay, F.; Kurata, M. Thermodynamic re-assessment of the Pu-U system and its application to the ternary Pu-U-Ga system. J. Nucl. Mater. 2014, 454, 81-95. [CrossRef]

66. Perron, A.; Turchi, P.E.A.; Landa, A.; Söderlind, P.; Ravat, B.; Oudot, B.; Delaunay, F. The Pu-U-Am system: An ab initio informed CALPHAD thermodynamic study. J. Nucl. Mater. 2015, 458, 425-441. [CrossRef]

67. Besmann, T.H.; Shin, D.; Lindemer, T.B. Uranium nitride as LWR TRISO fuel: Thermodynamic modeling of U-C-N. J. Nucl. Mater. 2012, 427, 162-168. [CrossRef]

68. Söderlind, P. First-principles elastic and structural properties of uranium metal. Phys. Rev. B 2002, 66, 085113. [CrossRef]

69. Sedmidubský, D.; Konings, R.J.M.; Novák, P. Calculation of enthalpies of formation of actinide nitrides. J. Nucl. Mater. 2005, 344, 40-44. [CrossRef]

70. Wyckoff, R.W.G. Crystal Structures; John Wiley \& Sons: New York, NY, USA, 1963; Volume I.

71. Mei, Z.-G.; Stan, M.; Pichler, B. First-principles study of structural, elastic, vibrational, and thermodynamic properties of UN. J. Nucl. Mater. 2013, 440, 63-69. [CrossRef]

72. Otero-de-la Roza, A.; Luaña, V. Gibbs2: A new version of the quasi-harmonic model code. I. Robust treatment of the static data. Comput. Phys. Commun. 2011, 182, 1708-1720. [CrossRef]

73. Otero-de-la Roza, A.; Abbasi-Pérez, D. Gibbs2: A new version of the quasi-harmonic model code. II Models for solid thermodynamics, features and implementation. Comput. Phys. Commun. 2011, 182, 2232-2248. [CrossRef]

74. Otero-de-la Roza, A.; Luaña, V. Equation of state and thermodynamics of solids using empirical corrections in the quasi-harmonic approximation. Phys. Rev. B 2011, 84, 184103. [CrossRef]

75. Otero-de-la Roza, A.; Luaña, V. Treatment of first-principles data for predictive quasiharmonic thermodynamics of solids: The case of MgO. Phys. Rev. B 2011, 84, 024109. [CrossRef]

76. Buyers, W.J.L.; Murray, A.F.; Holden, T.M.; Svensson, E.C.; DuPlessis, P.D.V.; Lander, G.H.; Vogt, O. Spin and phonon excitations in actinide systems. Physica 1980, 102B, 291-298. [CrossRef]

(C) 2019 by the authors. Licensee MDPI, Basel, Switzerland. This article is an open access article distributed under the terms and conditions of the Creative Commons Attribution (CC BY) license (http://creativecommons.org/licenses/by/4.0/). 